\title{
High-resolution mapping of the urban built environment stocks in Beijing
}

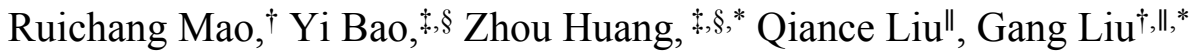

$\dagger$ SDU Life Cycle Engineering, Department of Chemical Engineering, Biotechnology, and Environmental Technology, University of Southern Denmark, 5230 Odense, Denmark

$\ddagger$ Institute of Remote Sensing and Geographical Information Systems, Peking University, Beijing, China

$\S$ Beijing Key Lab of Spatial Information Integration \& Its Applications, Peking University, Beijing, China

IIInstitute of Geographic Sciences and Natural Resources Research, Chinese Academy of Sciences, 100101 Beijing, China

\section{Corresponding Author}

*Phone: +86-10-62760132; e-mail: huangzhou@pku.edu.cn.

*Phone: +45-65009441; e-mail: gli@kbm.sdu.dk or geoliugang@gmail.com.

Supporting Information contains:

Number of pages: 23

Number of Tables: 14

Number of Figures: 18 


\section{Table of Content}

High-resolution mapping of the urban built environment stocks in Beijing.

1. Data collection and processing

2. Material Composition Indicators (MCIs) compiled from building samples and construction projects information (see Method section 2.3 in the main manuscript). S5

3. Building material stocks per age cohort. S9

4. Results of urban built environment material stocks of different cities. $\mathrm{S} 10$

5. The land use map of Beijing in $30 \mathrm{~m} \times 30 \mathrm{~m}$ resolution. S1 1

6. The quantity and composition of urban built environment stocks in Beijing in 2018. S12

7. The spatial distribution of built environment material stocks in Beijing in 2018.

8. Correlation between population density and built entrainment material stocks (MS) S19

9. Correlation between GDP and built environment material stocks. S21

10. References in the supporting information: S22 


\section{Data collection and processing}

Table S1. The carbon impact factor of different construction materials $\left(\mathrm{kg} \mathrm{CO}_{2-\mathrm{eq}} / \mathrm{t}\right)$.

\begin{tabular}{ccccccccc}
\hline Aluminum & Asphalt & Brick & Cement & Ceramic & Copper & Glass & Fly ash & Gravel \\
\hline 1650 & 221 & 271 & 843.25 & 857 & 2710 & 1136 & 107 & 1.45 \\
\hline Lime & Mineral Powder & Mortar & Sand & Steel & $\begin{array}{c}\text { Timbe } \\
\mathrm{r}\end{array}$ & Waterproof Paint \\
\hline 740 & 1.345 & 241 & 1.26 & 2655 & 300 & 2540 \\
\hline
\end{tabular}

Note:

The material carbon impact factors were collected from ecoinvent ${ }^{1}$ and eBalance ${ }^{2}$ database. Only fly ash and waterproof paint are found in ecoinvent database.

Table S2. The number of building samples across China (including Beijing) collected for the 12

\begin{tabular}{ccc}
\hline $\begin{array}{c}\text { Building } \\
\text { Typologies }\end{array}$ & $\begin{array}{c}\text { Number of } \\
\text { Building Samples }\end{array}$ & Percentage \\
\hline Agricultural & 21397 & $7.23 \%$ \\
Commercial & 24268 & $8.20 \%$ \\
Educational & 20771 & $7.02 \%$ \\
Historical & 19636 & $6.64 \%$ \\
Industrial & 14319 & $4.84 \%$ \\
Mixed & 3970 & $1.34 \%$ \\
Municipal & 8447 & $2.86 \%$ \\
Parking & 1513 & $0.51 \%$ \\
Public & 24739 & $8.36 \%$ \\
Residential & 151995 & $51.38 \%$ \\
Sport & 1484 & $0.50 \%$ \\
Storage & 3293 & $1.11 \%$ \\
\hline
\end{tabular}


Table S3. The research steps of deriving the age attribute for buildings.

1. Using the "Feature to Point" function in ArcGIS, the central points of building footprints were found.

2. Each building point (in step 1) had matched with the nearest residential/commercial point of interest (POI) data by using "Near" function, and assigned with a POI information.

3. Building age and corresponding location information were collected from the largest real-estate agency website in China (SouFun, http://soufun.com/) using a web-crawling approach.

4. Using the collected location information (step 3), we searched and matched the nearest surrounding POI by the "Near" function, and building point with POI was assigned with age information.

5. A K-nearest neighbors algorithm (KNN) method was used to supplement the missing information.

Table S4. The K-Nearest Neighbor method used for supplementing the age of buildings.

1. $\mathrm{k}$ is the number of nearest neighbors, $D$ is training set of building data

2. for every blank building data $\mathrm{z}=\left(\mathrm{x}^{\prime}, \mathrm{y}^{\prime}\right)$ do

3. Calculating the distance $\mathbb{d}\left(\mathrm{x}^{\prime}, \mathrm{x}\right)$ between $\mathrm{z}$ and sample $(\mathrm{x}, \mathrm{y}) \in D, \mathrm{x}$ is the building location, $\mathrm{y}$ is building attribute, $\mathbb{d}(\cdot)$ is the Euclidean distance between buildings

4. Choosing the set $D_{z}$ which is nearest to $\mathrm{z}$ and the size of $D_{z}$ is $\mathrm{k}$, and $D_{z}$ $\subseteq D$

5. $\mathrm{y}^{\prime}={ }_{v}^{\operatorname{argmax}} \sum_{\left(x_{i}, y_{i}\right) \in D} I\left(v=y_{i}\right)$

6. end for 
Table S5. Data description, applications, and the sources in this study.

\begin{tabular}{|c|c|c|c|}
\hline Types of data & Description of data & Application for & Sources \\
\hline Building & $\begin{array}{l}295896 \text { buildings in a } \\
\text { rectangle area from } \\
116.1605,39.7649 \text { to } \\
116.5701,40.0413 \text { in } \\
\text { Beijing. }\end{array}$ & $\begin{array}{l}\text { Building stock } \\
\text { calculation. }\end{array}$ & $\begin{array}{l}\text { AutoNavi } \\
\text { Company }\end{array}$ \\
\hline Infrastructure & $\begin{array}{l}\text { Road, railway and metro } \\
\text { location, width, road width } \\
\text { and types, and metro station } \\
\text { location. }\end{array}$ & $\begin{array}{l}\text { Infrastructure stock } \\
\text { calculation. }\end{array}$ & $\begin{array}{l}\text { AutoNavi } \\
\text { Company }\end{array}$ \\
\hline Land Use & $\begin{array}{l}11 \text { categories of land use in } \\
30 \mathrm{~m} \times 30 \mathrm{~m} \text { spatial } \\
\text { resolution. }\end{array}$ & $\begin{array}{l}\text { Identifying the } \\
\text { typology of buildings. }\end{array}$ & $\begin{array}{l}\text { Planning } \\
\text { Administration of } \\
\text { Beijing }\end{array}$ \\
\hline POI & $\begin{array}{l}\text { The residential and } \\
\text { commercial POI. }\end{array}$ & $\begin{array}{l}\text { Identifying the age of } \\
\text { buildings. }\end{array}$ & $\begin{array}{l}\text { AutoNavi } \\
\text { Company }\end{array}$ \\
\hline $\begin{array}{l}\text { SouFun website } \\
\text { data }\end{array}$ & $\begin{array}{l}\text { Relatively complete age } \\
\text { information of buildings. }\end{array}$ & $\begin{array}{l}\text { Identifying the age of } \\
\text { buildings. }\end{array}$ & $\begin{array}{l}\text { SouFun, } \\
\text { http://soufun.com/ }\end{array}$ \\
\hline
\end{tabular}


2. Material Composition Indicators (MCIs) compiled from building samples and construction projects information (see Method section 2.3 in the main manuscript).

Table S6a. Detailed MCIs for buildings above ground constructed before 1980 in Beijing.

\begin{tabular}{|c|c|c|c|c|c|c|c|c|c|c|c|}
\hline $\begin{array}{l}\text { Surface Building before } \\
1980\left(\mathrm{~kg} / \mathrm{m}^{2}\right)\end{array}$ & Cement & Steel & Timber & Brick & Gravel & Sand & Asphalt & Lime & Glass & Ceramic & Total \\
\hline Historical & 27 & 1 & 88 & 52 & 269 & 10 & 0 & 4 & 1 & 18 & 470 \\
\hline Commercial & 399.92 & 68.62 & 10.28 & 443.63 & 800.6 & 811.78 & 4.51 & 43.69 & 1.71 & 0 & 2584.74 \\
\hline Municipal & 222 & 35.75 & 28.44 & 663.92 & 557.71 & 528.61 & 2.35 & 39.39 & 2.3 & 0 & 2080.47 \\
\hline Storage & 1.88 & 0 & 0 & 229.50 & 0 & 0 & 0 & 0 & 0 & 0 & 231.38 \\
\hline Mixed & 404.08 & 93.51 & 6.93 & 289.65 & 680.95 & 566.74 & 1.72 & 49.98 & 1.07 & 0 & 2094.63 \\
\hline Industrial & 218.43 & 50.65 & 2.08 & 595.08 & 275.20 & 272.68 & 2.52 & 9.48 & 2.77 & 0 & 1428.89 \\
\hline Public & 274.88 & 54.92 & 11.63 & 728.95 & 503.72 & 619.33 & 2.67 & 30.81 & 2.06 & 0 & 2228.97 \\
\hline Educational & 187.68 & 25.44 & 14.79 & 854.28 & 543.95 & 604.44 & 2.59 & 55.45 & 2.68 & 0 & 2291.3 \\
\hline Residential & 20 & 0 & 7 & 850 & 0 & 256 & 0 & 0 & 0 & 0 & 1133 \\
\hline Sport & 209.68 & 40.35 & 52.57 & 612.28 & 889.34 & 717.15 & 5.24 & 42.39 & 2.77 & 0 & 2571.77 \\
\hline Parking & 87.5 & 8.25 & 1.05 & 416.50 & 0 & 0 & 0 & 0 & 0 & 0 & 513.3 \\
\hline Agricultural & 16.67 & 0 & 0.43 & 498.67 & 25 & 50 & 0 & 0 & 0 & 0 & 590.77 \\
\hline
\end{tabular}


Table S6b. Detailed MCIs for buildings above ground constructed from 1981 to 2000 in Beijing.

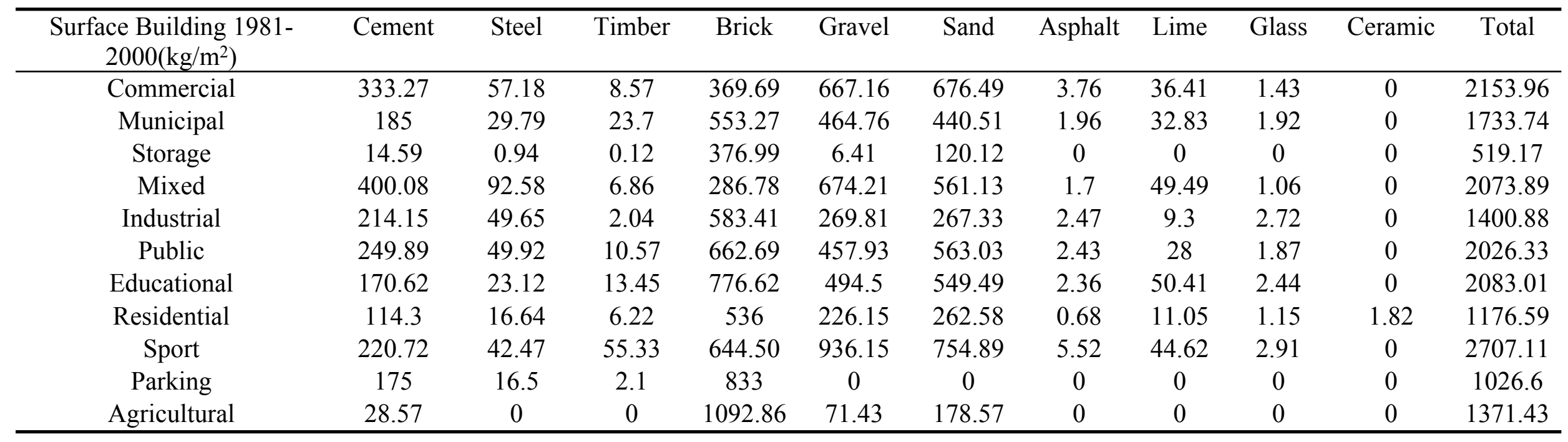


Table S6c. Detailed MCIs for buildings above ground constructed from 2001 to 2018 in Beijing.

\begin{tabular}{|c|c|c|c|c|c|c|c|c|c|c|c|}
\hline $\begin{array}{l}\text { Surface Building 2001- } \\
2018\left(\mathrm{~kg} / \mathrm{m}^{2}\right)\end{array}$ & Cement & Steel & Timber & Brick & Gravel & Sand & Asphalt & Lime & Glass & Ceramic & Total \\
\hline Commercial & 206.68 & 133.23 & 2.38 & 88.64 & 500.49 & 329.09 & 0 & 0 & 0 & 3.4 & 1263.91 \\
\hline Municipal & 138.48 & 70.72 & 0 & 0 & 370.09 & 421 & 0.13 & 0 & 0 & 0 & 1000.42 \\
\hline Storage & 45.45 & 0 & 0 & 185.45 & 65.91 & 349.09 & 0 & 0 & 0 & 0 & 645.9 \\
\hline Mixed & 146.95 & 71.11 & 1.28 & 263.5 & 0.13 & 23.06 & 0 & 0 & 0 & 0 & 506.03 \\
\hline Industrial & 154.73 & 50.48 & 6.05 & 188.26 & 216.67 & 144.40 & 0.24 & 0 & 0 & 0 & 760.83 \\
\hline Public & 183.86 & 92.44 & 1.96 & 373.29 & 456.80 & 301.67 & 0.09 & 0 & 0 & 2.05 & 1412.16 \\
\hline Educational & 215.99 & 50.64 & 0 & 0 & 636.63 & 614.64 & 0.1 & 0 & 0 & 0 & 1518 \\
\hline Residential & 84.66 & 25.85 & 9.27 & 319.47 & 192.30 & 214.52 & 0.02 & 0 & 0.05 & 0.45 & 846.59 \\
\hline Sport & 241.48 & 80.63 & 2.08 & 177.46 & 536.42 & 315.5 & 0.36 & 0 & 0.07 & 1.19 & 1355.19 \\
\hline Parking & 367.46 & 95.63 & 0.26 & 118.58 & 1438.81 & 887.8 & 1.41 & 0 & 0 & 0 & 2909.95 \\
\hline Agricultural & 37.38 & 0 & 0.53 & 842.31 & 11.07 & 14.05 & 0 & 0 & 0 & 0 & 905.34 \\
\hline
\end{tabular}

Table S6d. Detailed MCIs for buildings under ground in Beijing.

\begin{tabular}{ccccccccccccc}
\hline Subsurface Building $(\mathrm{kg} / \mathrm{m} 2)$ & Cement & Steel & Brick & $\begin{array}{c}\text { Waterproof } \\
\text { paint }\end{array}$ & Mortar & Gravel & Lime & Asphalt & Sand & Total \\
\hline Basement & 435.06 & 0.03 & 18.67 & 0.29 & 0.36 & 0 & 0 & 0 & 0 & 454.4 \\
Foundation & 51.16 & 0 & 0 & 0 & 0 & 139.17 & 0 & 0 & 56.79 & 247.12 \\
Garage & 500 & 20 & 0.14 & 0.01 & 0.15 & 19.52 & 13.1 & 1.86 & 0 & 554.77 \\
\hline
\end{tabular}


Table S6e. Detailed MCIs for different types of roads in Beijing.

\begin{tabular}{cccccccc}
\hline Road (ton/km) & Cement & Steel & Gravel & Sand & Asphalt & Mineral Powder & Total \\
\hline Expressway & 1102.35 & 0 & 73046.9 & 3625.08 & 1250.26 & 1184.64 & 80209.24 \\
First & 826.76 & 0 & 54785.18 & 2718.81 & 937.7 & 888.48 & 60156.93 \\
Second & 661.41 & 0 & 43828.14 & 2175.05 & 750.16 & 710.79 & 48125.54 \\
Third & 2685.26 & 41.73 & 23234.5 & 2615.71 & 2.74 & 0 & 28579.94 \\
Fourth & 1195.84 & 18.58 & 10347.1 & 1164.86 & 1.22 & 0 & 12727.6 \\
\hline
\end{tabular}

Table S6f. Detailed MCIs for railways in Beijing.

\begin{tabular}{llllllllll}
\hline Railway (ton/km) & Cement & Steel & Gravel & Sand & Lime & Fly ash & Copper & Aluminum & Total \\
\hline Ballastless track & 515 & 300 & 1500 & 1180 & 0 & 120 & 0 & 0 & \\
Foundation & 3525 & 0 & 12450 & 5350 & 1525 & 89 & 0 & 0 & 26679 \\
Electric, communication, and signaling systems & 0 & 50 & 0 & 0 & 0 & 50 & 13 & 12 & \\
\hline
\end{tabular}

Table S6g. Detailed MCIs for metro in Beijing.

\begin{tabular}{cccccccccccc}
\hline Metro & \multirow{2}{*}{ Cement } & \multirow{2}{*}{ Steel } & \multirow{2}{*}{ Gravel } & Sand & Asphalt & Copper & Rubber & Geotextile & PVC & W & Total \\
\hline Lines (ton $/ \mathrm{km})$ & 18384.99 & 114 & & & & & 0.03 & 0.04 & 0.44 & 19529 \\
Station (ton) & 210554.7 & 16278.1 .7 & 678.9 & 4366 & 3037.44 & 4.54 & 6.10 & 1.32 & & 94.04 & 235014 \\
\hline
\end{tabular}




\section{Building material stocks per age cohort.}

Table S7. Number of buildings, floor areas, and building material stocks per age cohort in Beijing.

\begin{tabular}{ccccccccc}
\hline Age cohort & $\begin{array}{c}\text { Before } \\
1980\end{array}$ & 1980 'S & $\begin{array}{c}1991- \\
1995\end{array}$ & $\begin{array}{c}1996- \\
2000\end{array}$ & $\begin{array}{c}2001- \\
2005\end{array}$ & $\begin{array}{c}2006- \\
2010\end{array}$ & $\begin{array}{c}2011- \\
2015\end{array}$ & $\begin{array}{c}2016- \\
2018\end{array}$ \\
\hline $\begin{array}{c}\text { Number of } \\
\text { buildings }\end{array}$ & 25921 & 10636 & 13116 & 18996 & 33965 & 129688 & 54931 & 26731 \\
\hline Floor Areas $\left(\mathrm{km}^{2}\right)$ & 18.87 & 15.84 & 19.05 & 74.44 & 78.47 & 43.25 & 22.83 & 3.89 \\
\hline Building MS (Mt) & 57 & 168 & 154 & 671 & 700 & 350 & 150 & 20 \\
\hline
\end{tabular}




\section{Results of urban built environment material stocks of different cities.}

Table S8. Comparision of urban built environment material stocks among different case cities.

\begin{tabular}{|c|c|c|c|c|}
\hline $\begin{array}{l}\text { System } \\
\text { boundary }\end{array}$ & Case city & $\begin{array}{l}\text { Stock per } \\
\text { capita (ton } \\
\text { /cap) }\end{array}$ & $\begin{array}{l}\text { Stock } \\
\text { Density } \\
\left(\mathrm{kt} / \mathrm{km}^{2}\right)\end{array}$ & Reference \\
\hline \multirow{3}{*}{$\begin{array}{l}\text { Building \& } \\
\text { Infrastructure }\end{array}$} & Beijing & 140 & 1457 & \multirow{3}{*}{$\begin{array}{l}\text { Our Study } \\
\text { Tanikawa, Hiroki, and Seiji } \\
\text { Hashimoto. }(2009)^{3} \\
\text { Tanikawa, Hiroki, and Seiji } \\
\text { Hashimoto. }(2009)^{3}\end{array}$} \\
\hline & $\begin{array}{l}\text { Wakayama } \\
\text { City }\end{array}$ & 247 & 1118 & \\
\hline & $\begin{array}{l}\text { Salford } \\
\text { Quays }\end{array}$ & 111 & 267 & \\
\hline \multirow{7}{*}{ Building } & Beijing & 121 & 1259 & \multirow{7}{*}{$\begin{array}{l}\text { Our Study } \\
\text { Chen, Chang, et al. (2016) } \\
\text { Cheng, Kuang-Ly, et al. (2018) } \\
\text { Marcellus-Zamora, Kimberlee A., et } \\
\text { al. (2016) }{ }^{6} \\
\text { Kleemann, Fritz, et al. }(2017)^{7} \\
\text { Miatto, Alessio, et al. }(2019)^{8} \\
\text { Stephan, André, and Aristide } \\
\text { Athanassiadis. }(2017)^{9}\end{array}$} \\
\hline & Ezhou & 47 & 569 & \\
\hline & Taipei & 68 & 675 & \\
\hline & Philadelphia & NA & 1369 & \\
\hline & Vienna & 210 & 960 & \\
\hline & Padua & 209 & 464 & \\
\hline & Melbourne & NA & 904 & \\
\hline \multirow{3}{*}{$\begin{array}{l}\text { Residential } \\
\text { Building }\end{array}$} & Beijing & 63 & 659 & \multirow{3}{*}{$\begin{array}{l}\text { Our Study } \\
\text { Han, Ji, et al. (2018) } \\
\text { Mesta, Carlos, Ramzy Kahhat, and } \\
\text { Sandra Santa-Cruz. (2019) }\end{array}$} \\
\hline & Shanghai & 22 & 84 & \\
\hline & Chiclayo & 55 & 608 & \\
\hline
\end{tabular}

\section{The land use map of Beijing in $30 \mathrm{~m} \times 30 \mathrm{~m}$ resolution.}

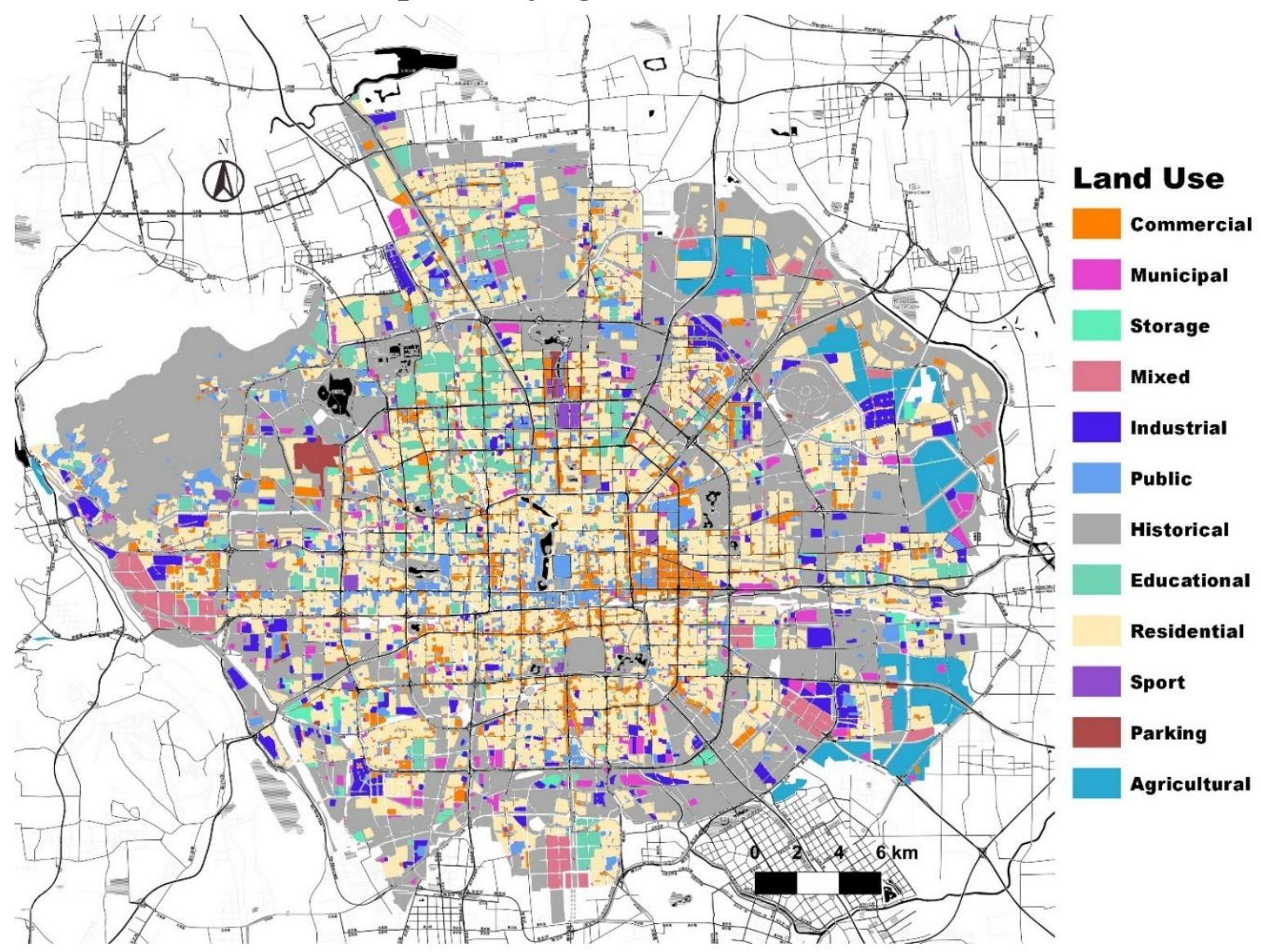

Figure S1. The land use map of Beijing in $30 \mathrm{~m} \times 30 \mathrm{~m}$ resolution with 12 detailed typologies. 
6. The quantity and composition of urban built environment stocks in Beijing in 2018.

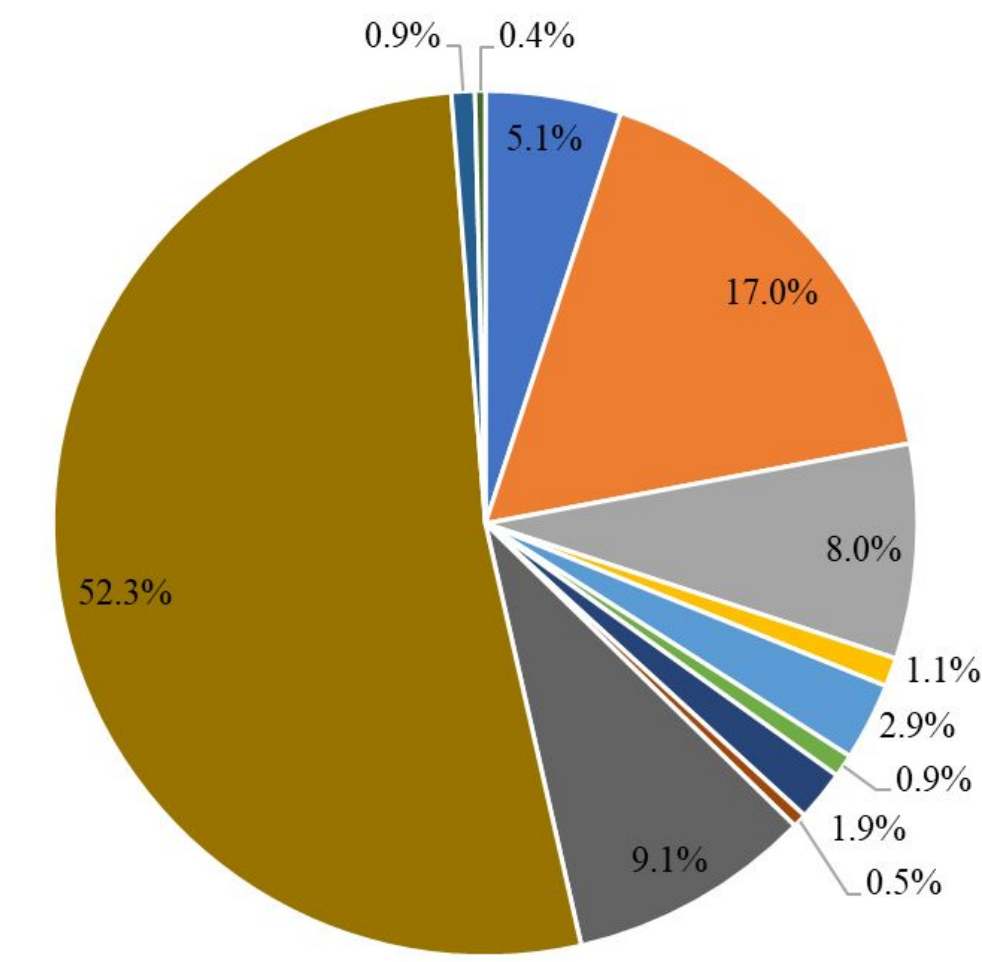

\begin{tabular}{|c|c|c|c|}
\hline $\begin{array}{l}\text { Agricultural } \\
\text { - Industrial } \\
\text { - Public }\end{array}$ & $\begin{array}{l}\text { - Commercial } \\
\text { - Mixed } \\
\text { - Residential }\end{array}$ & $\begin{array}{l}\text { Educational } \\
\text { - Municipal } \\
\text { - Sport }\end{array}$ & $\begin{array}{l}\text { Historical } \\
\text { - Parking } \\
\text { - Storage }\end{array}$ \\
\hline
\end{tabular}

Figure S2. The composition of building material stocks by building topology in Beijing. 


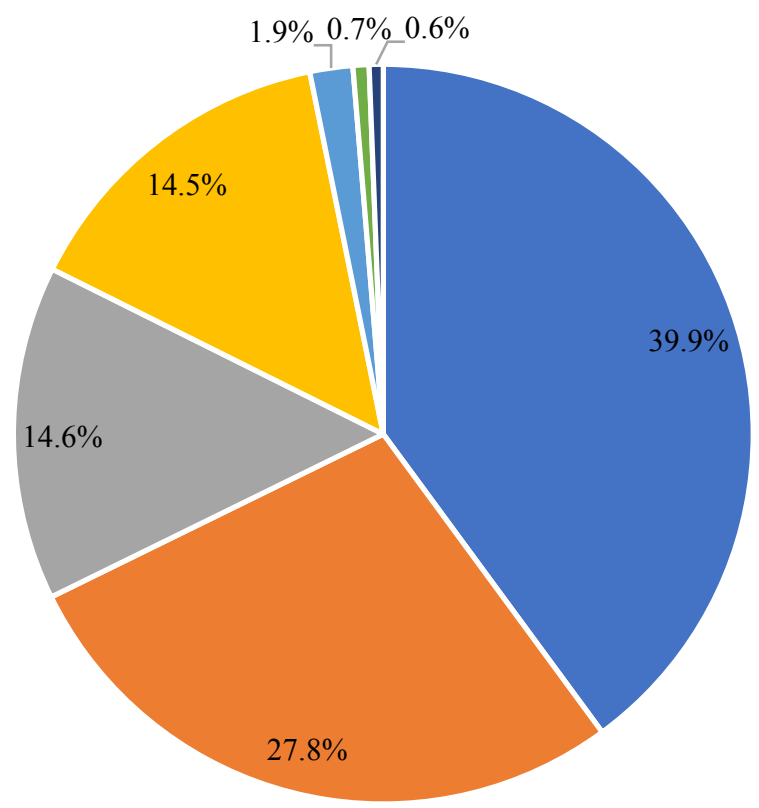

Gravel $\square$ Cement $\square$ Brick $\square$ Sand $\square$ Steel $\square$ Lime $\square$ Others

Figure S3. The composition of built environment stocks by material in Beijing.

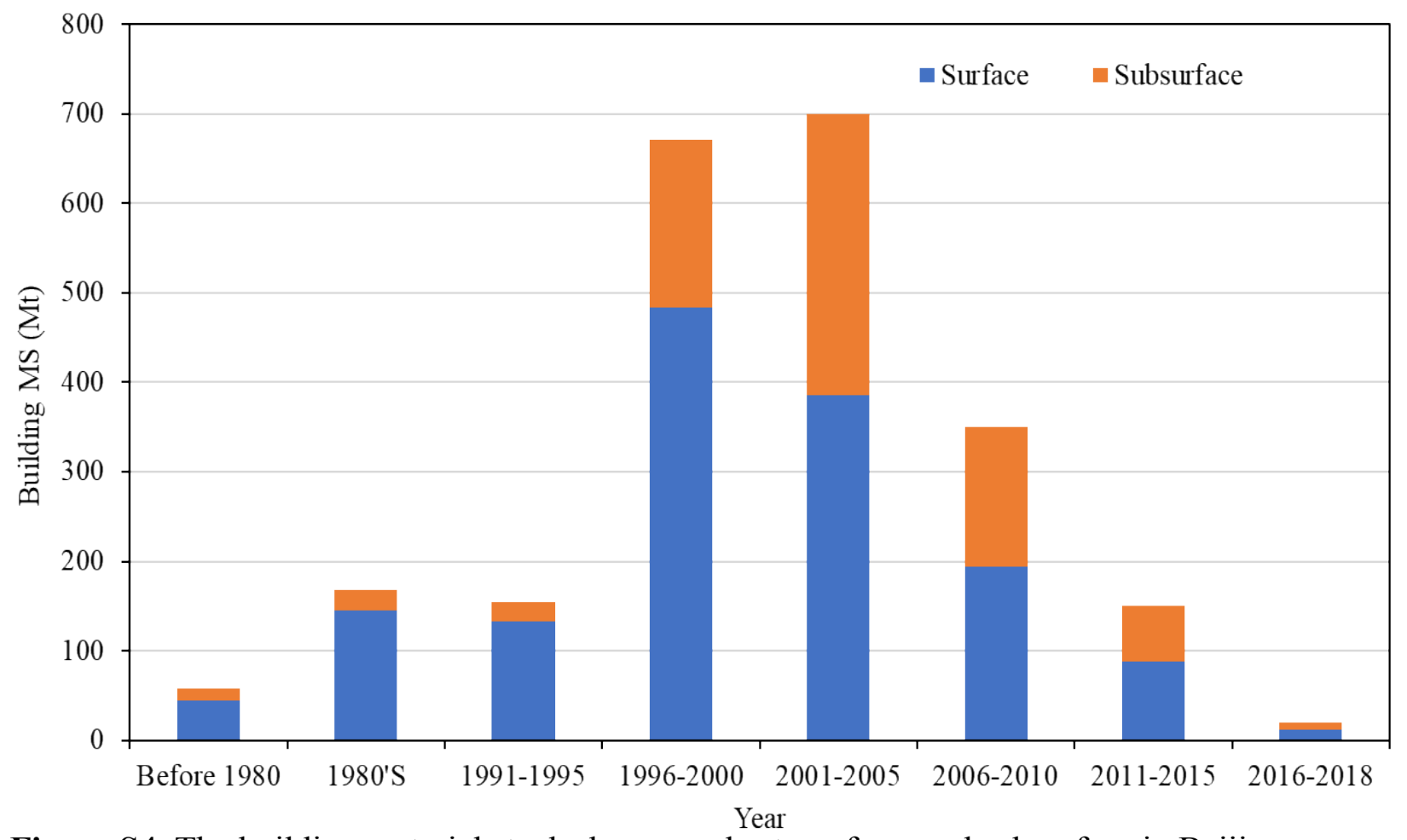

Figure S4. The building material stocks by age cohort, surface, and subsurface in Beijing. 


\section{The spatial distribution of built environment material stocks in Beijing in}

2018.
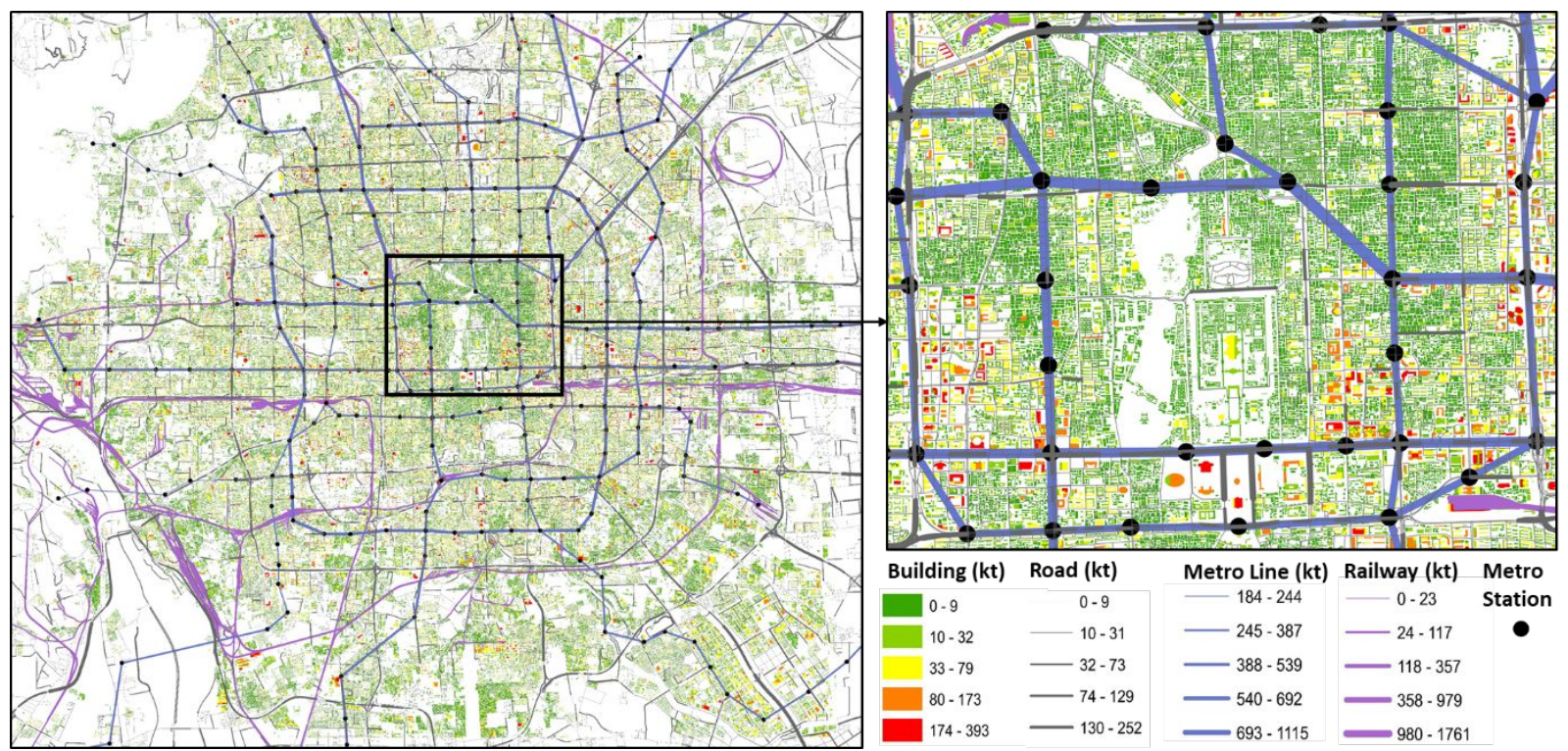

Figure S5. The spatial distribution of built environment material stocks in Beijing in 2018. 


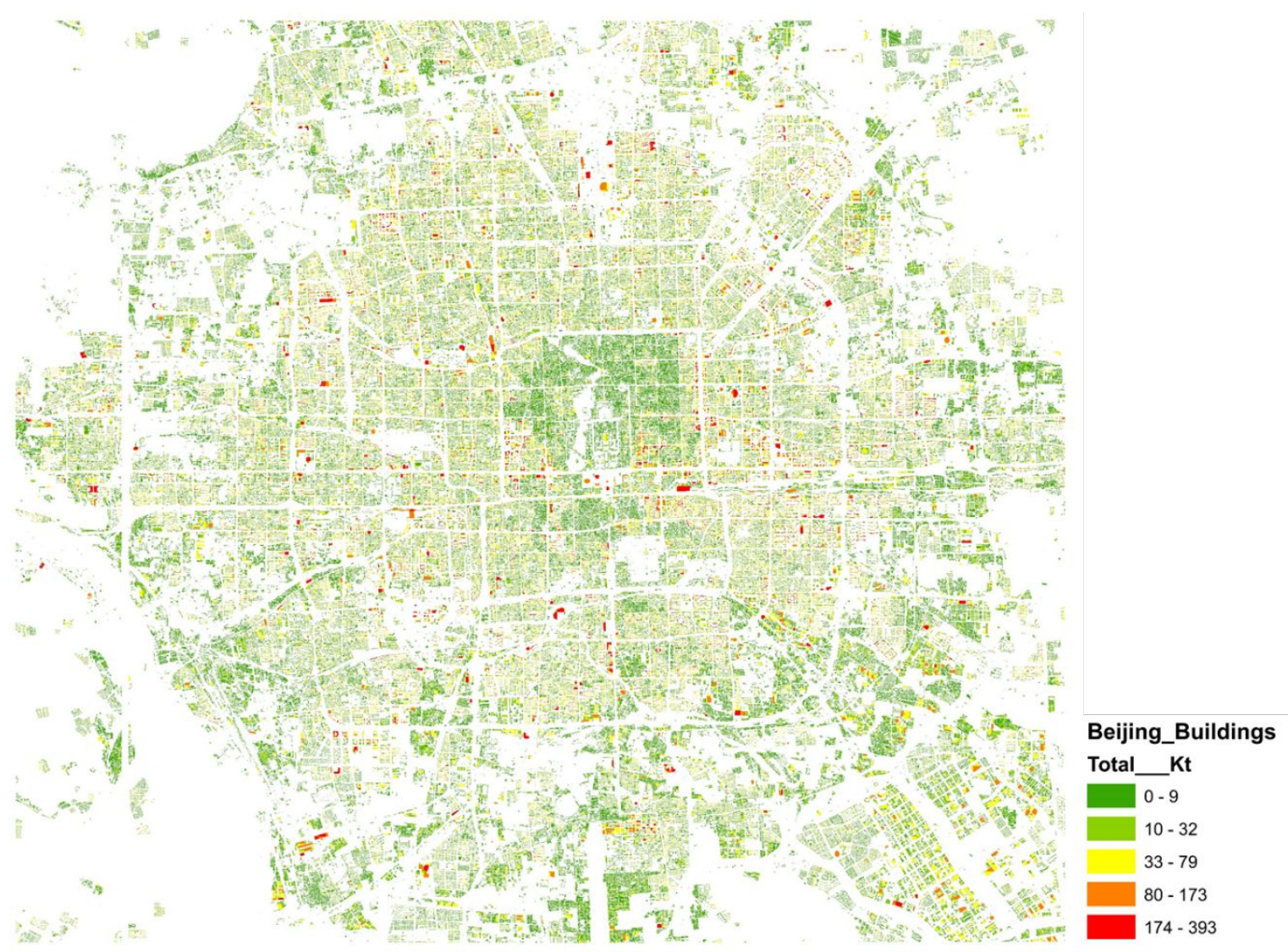

Figure S6. The spatial distribution of building material stocks in Beijing in 2018.

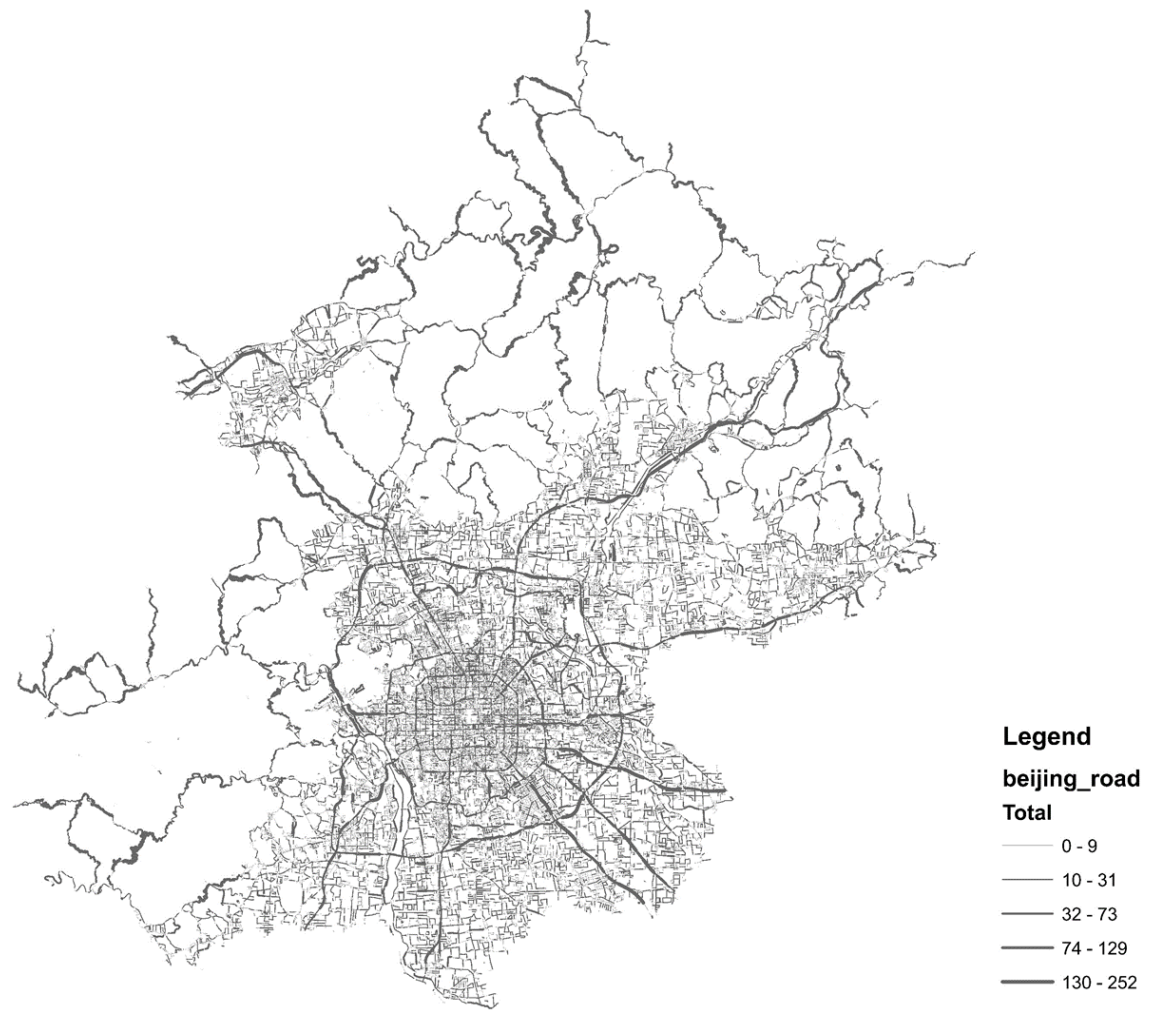

Figure S7. The spatial distribution of road material stocks in Beijingin 2018. 

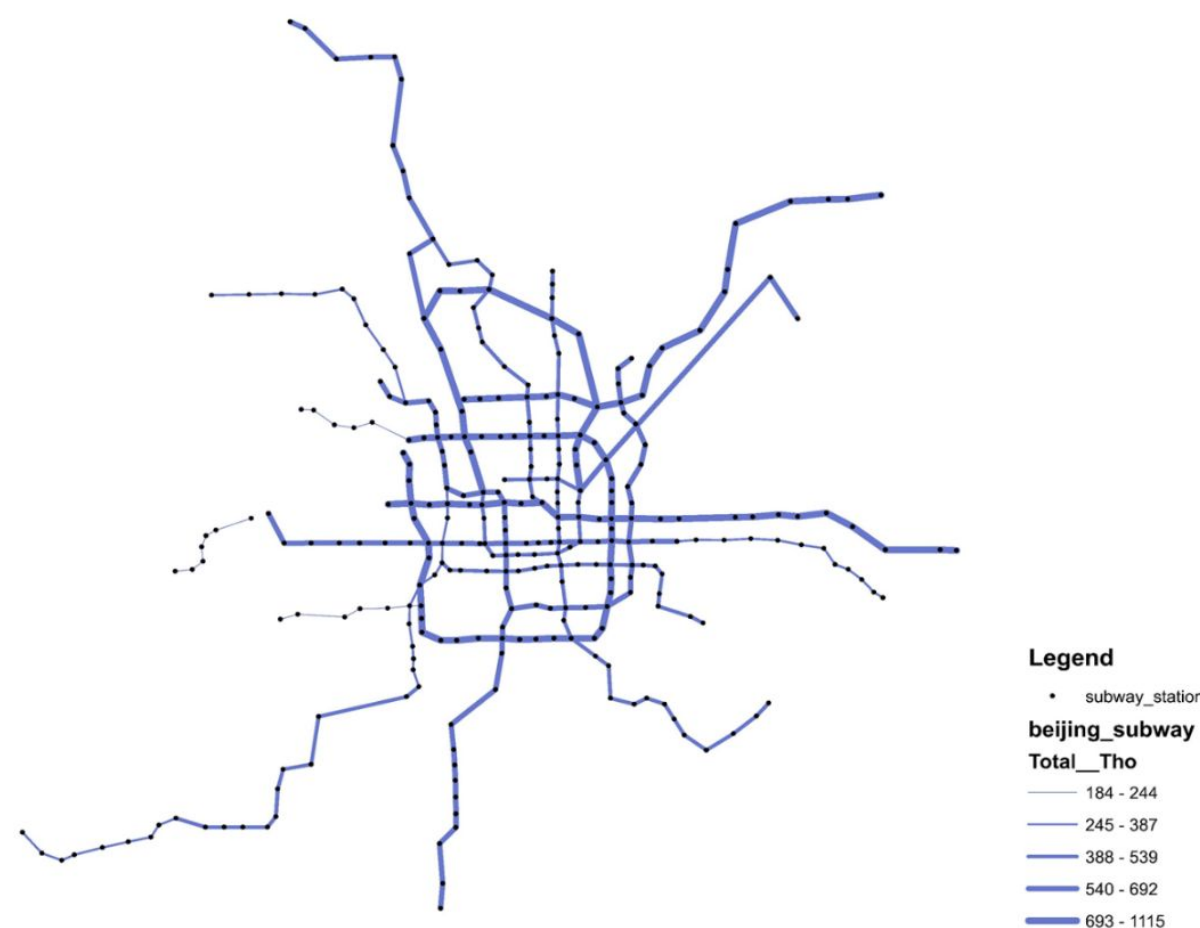

Figure S8. The spatial distribution of metro material stocks in Beijing in 2018.
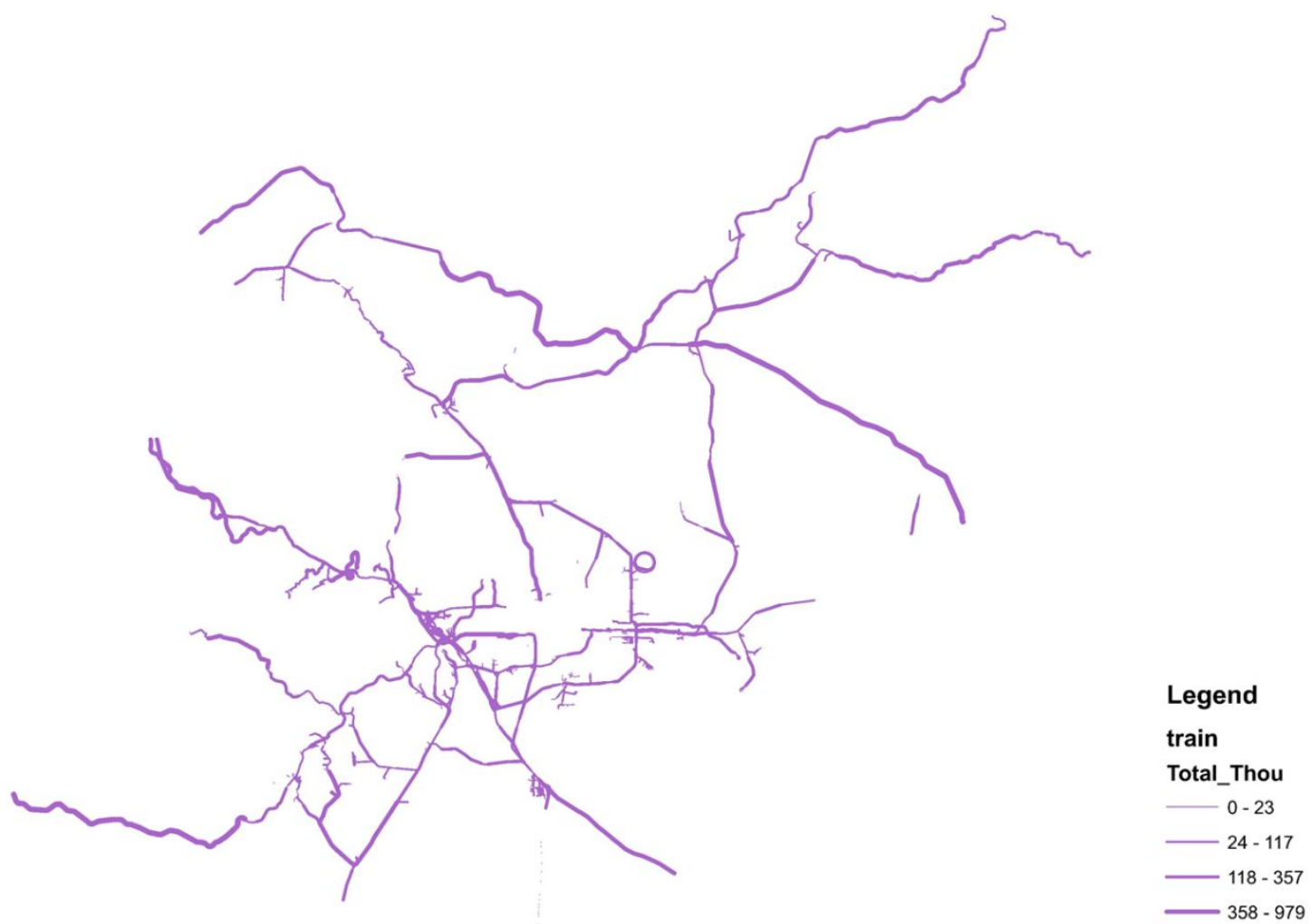

Figure S9. The spatial distribution of railway material stocks in Beijing in 2018. 


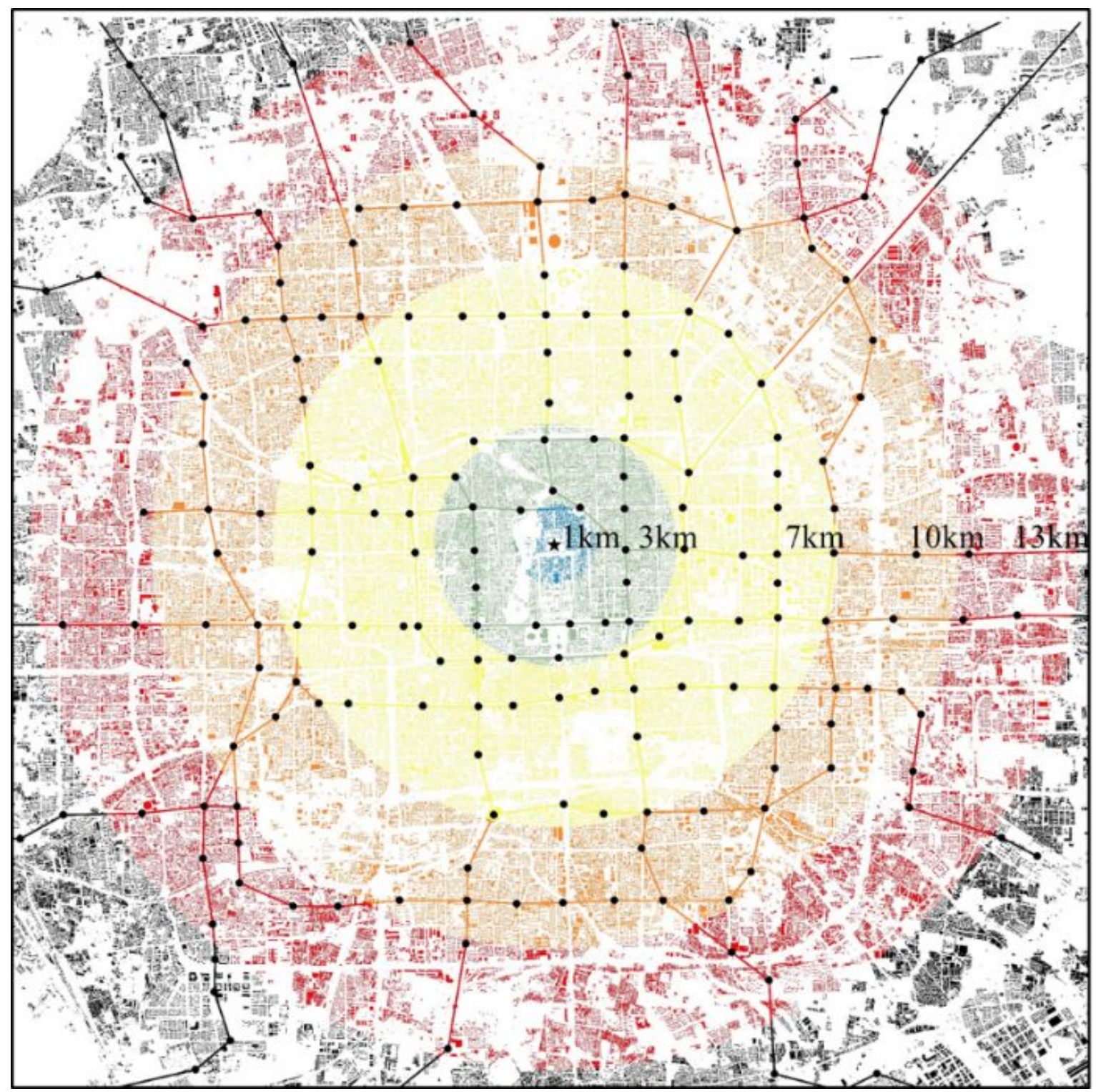

Figure S10. The distribution of built environment material stocks in different belts from city center (0-1 km, 1-3 km, 3-7 km, 7-10 km, and 10-13km) in Beijing. 

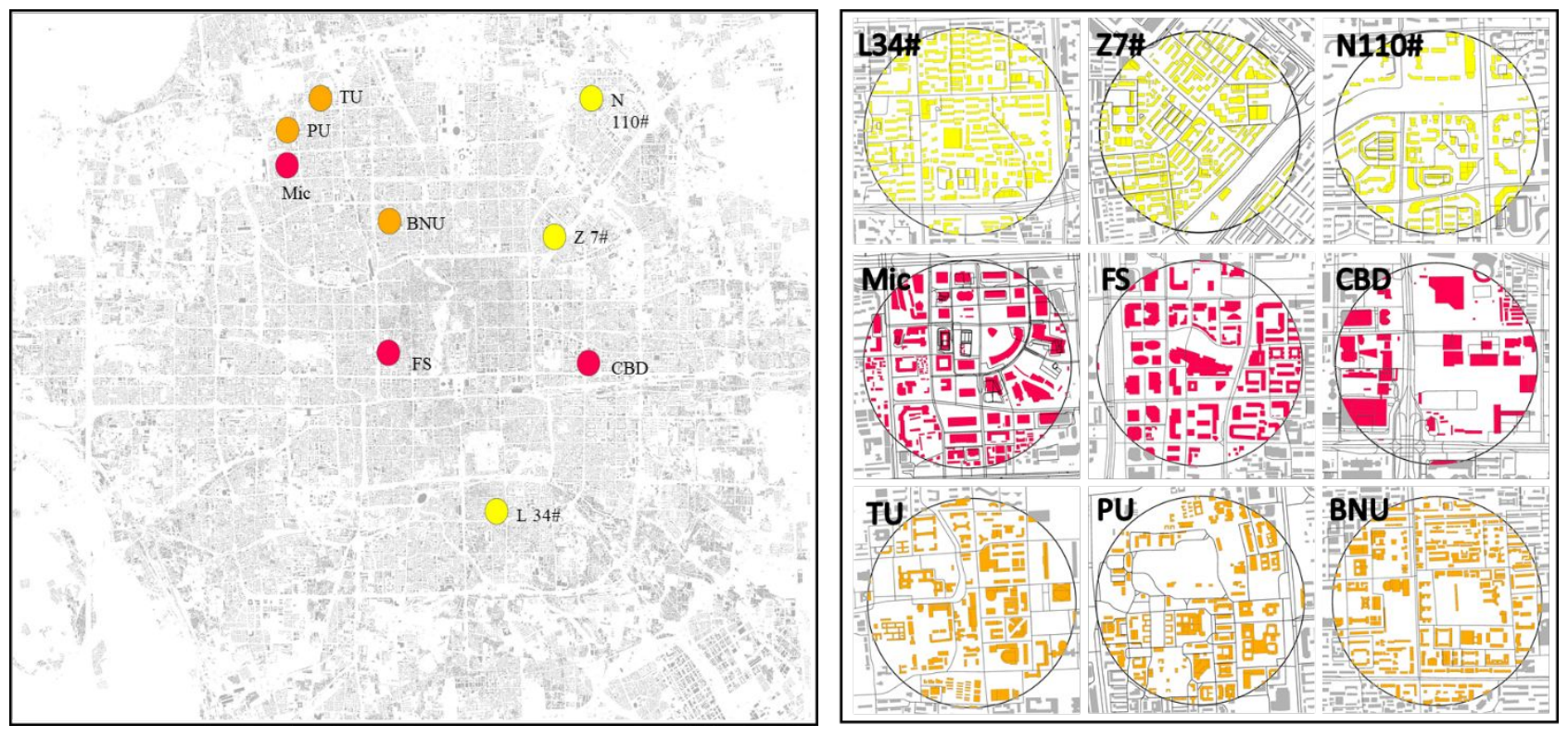

Figure S11. The built environment material stocks of 9 selected case areas in 3 typologies in a $500 \mathrm{~m}$ 's radius circle in Beijing.

Note: The yellow circles are residential sites, the red circles are commercial, and the orange circles are educational. The radius of the circle is $500 \mathrm{~m}$ and all building and infrastructure stocks are included. Material stock volume and density in commercial sites (10.2 Mt and $12.9 \mathrm{t} / \mathrm{m} 2)$ were around 2 times of those in residential sites (5.3 Mt and $6.8 \mathrm{t} / \mathrm{m} 2)$, and 3.5 times of those in educational sites (2.9 Mt and $3.7 \mathrm{t} / \mathrm{m} 2)$. 


\section{Correlation between population density and built entrainment material stocks (MS).}

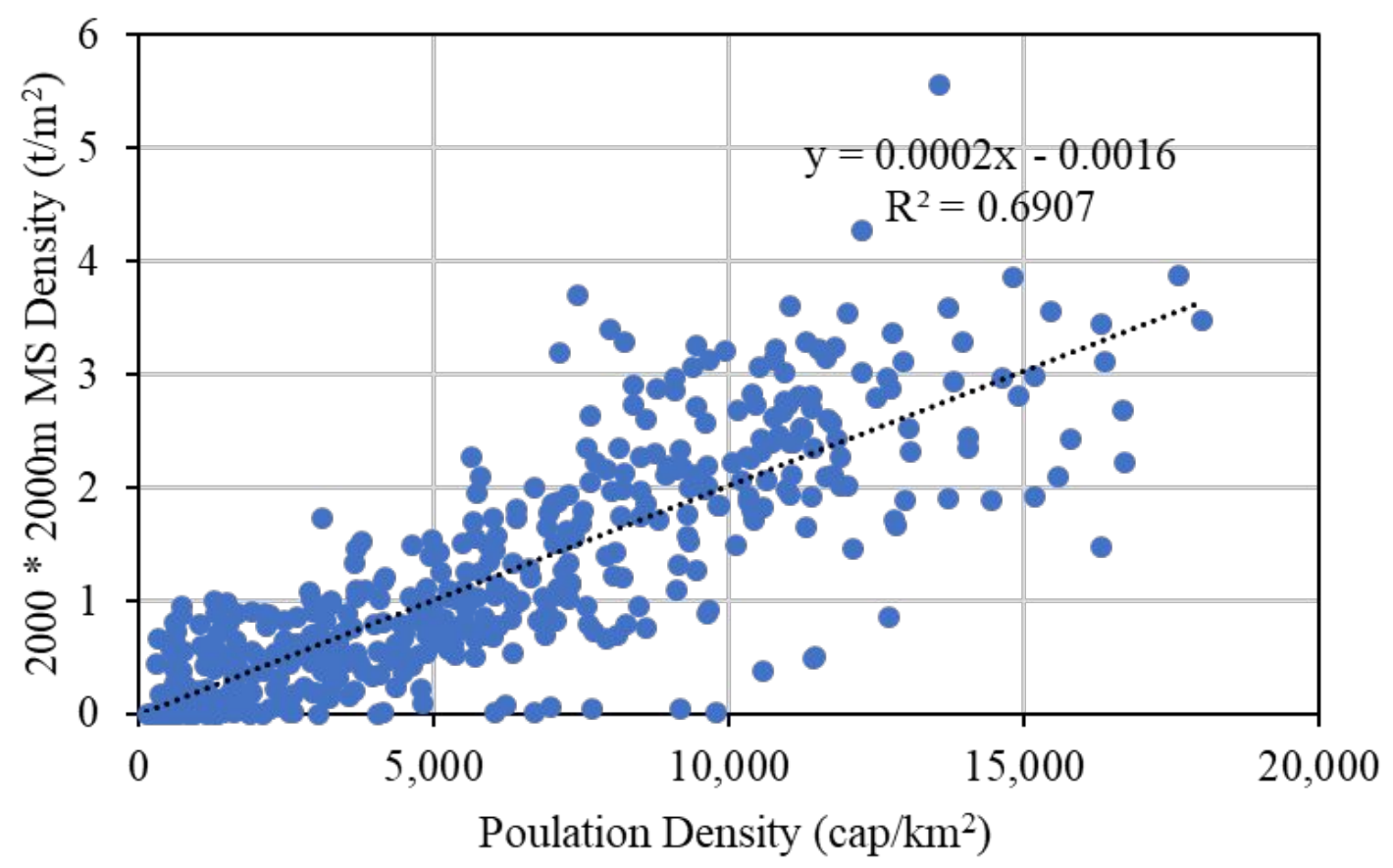

Figure S12. Correlation between population density and built entrainment material stocks density (in $2000 * 2000 \mathrm{~m}$ resolution) in Beijing.

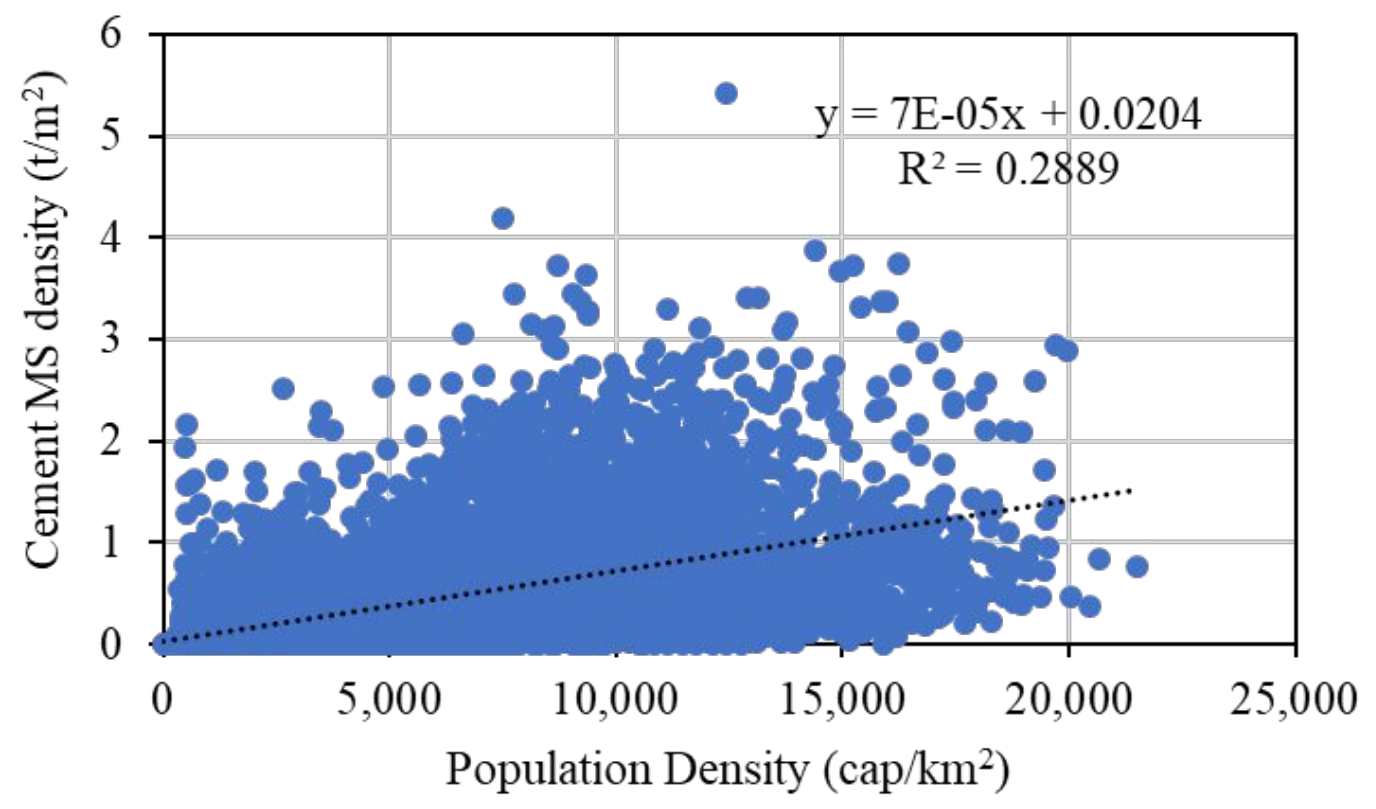

Figure S13. Correlation between population density and cement material stocks density in Beijing. 


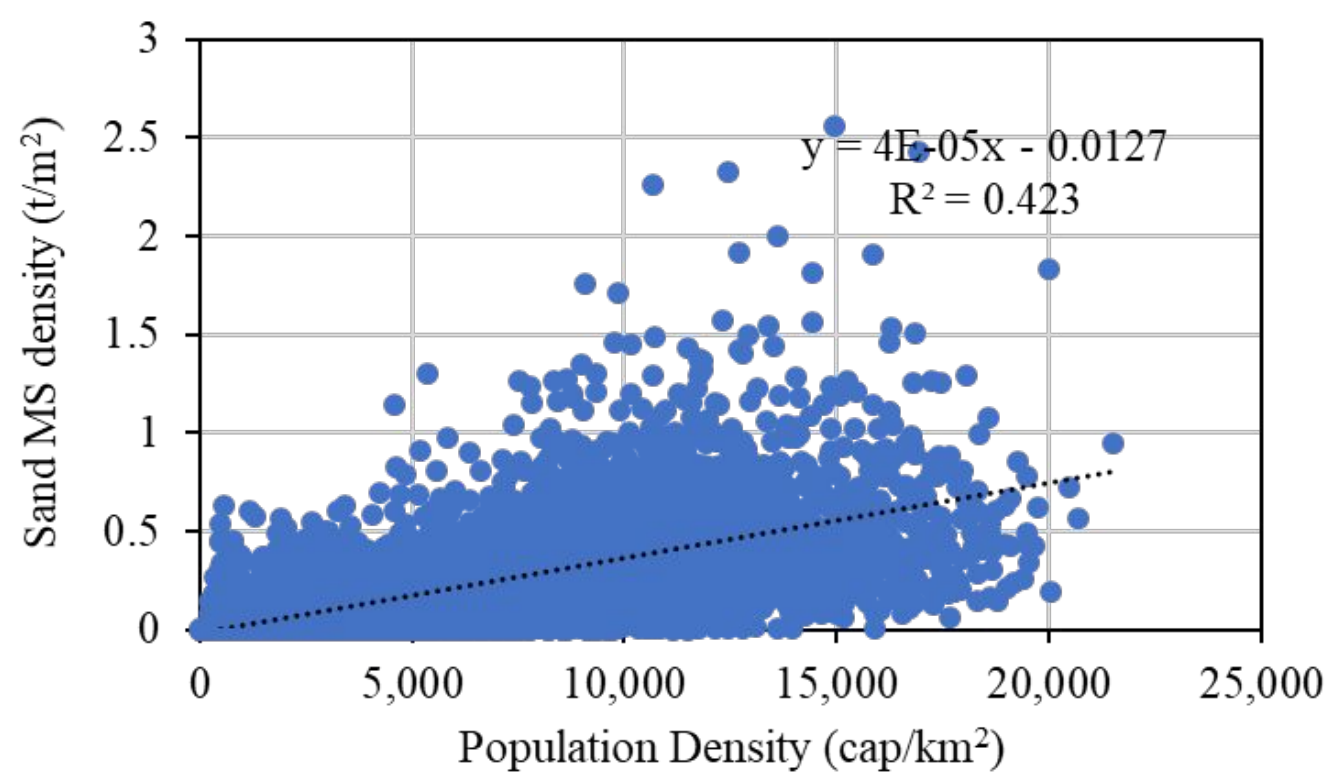

Figure S14. Correlation between population density and sand material stocks density in Beijing.

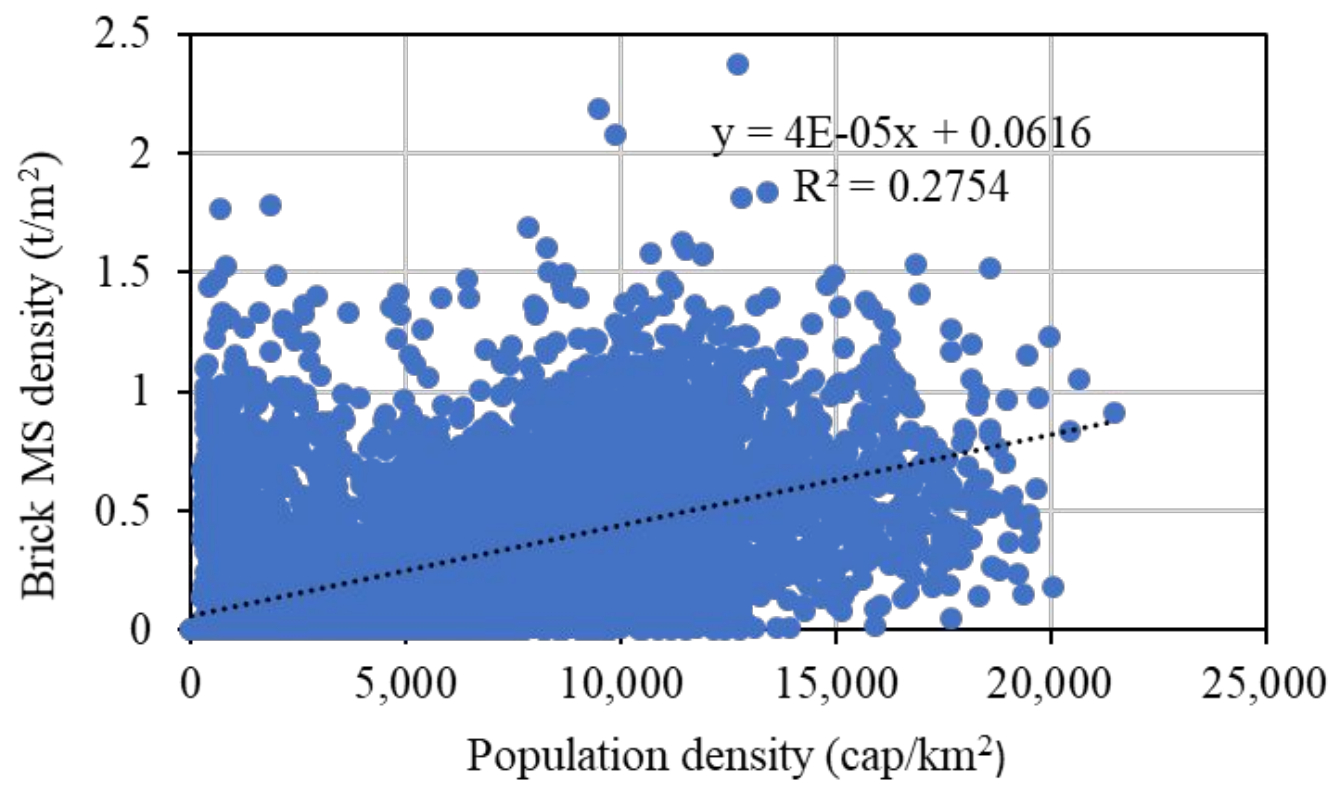

Figure S15. Correlation between population density and brick material stocks density in Beijing. 


\section{Correlation between GDP and built environment material stocks.}

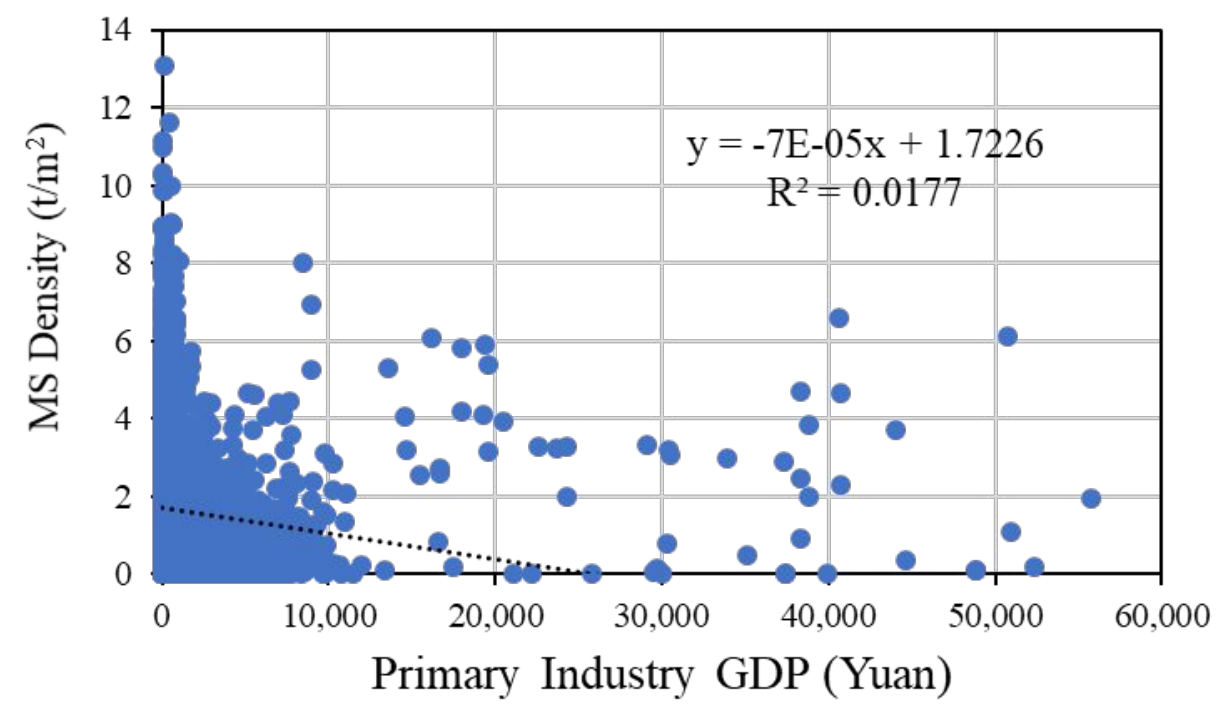

Figure S16. Correlation between Primary Industry GDP and built environment material stocks density in Beijing.

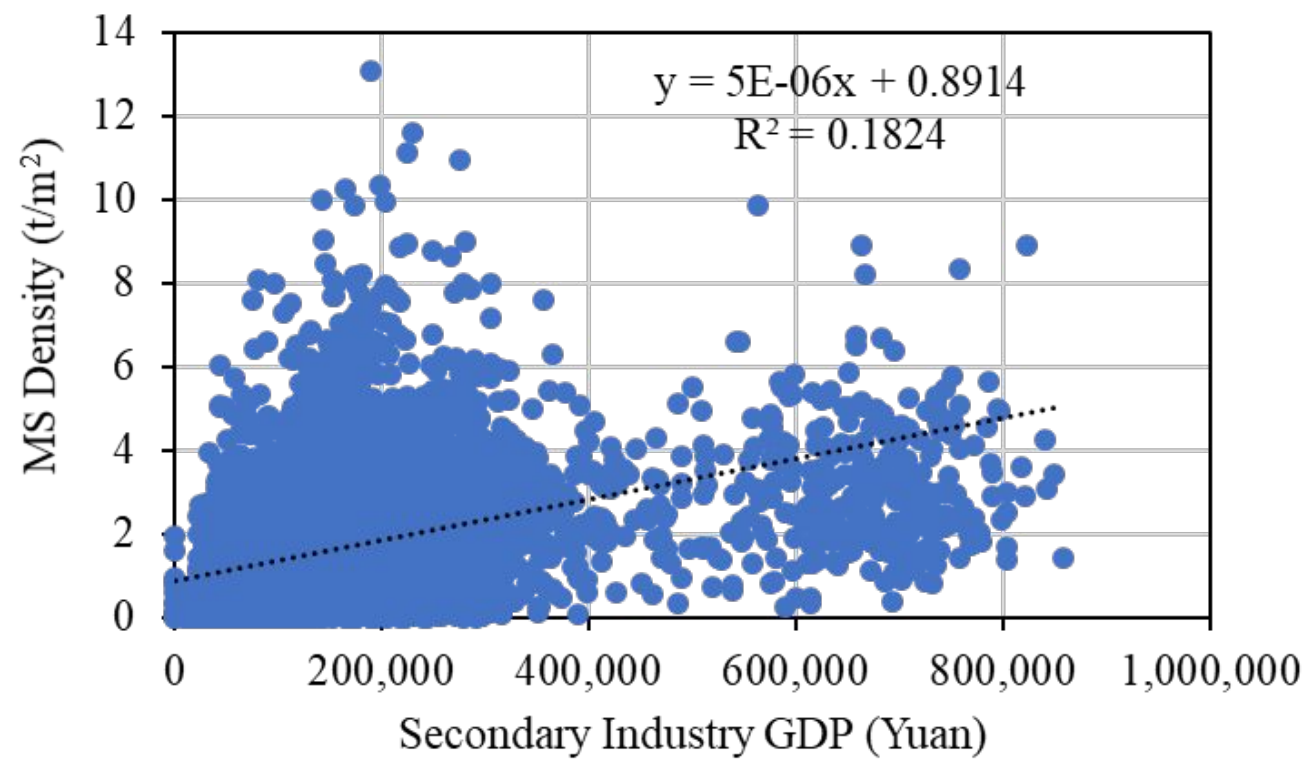

Figure S17. Correlation between Secondary Industry GDP and built environment material stocks density in Beijing. 


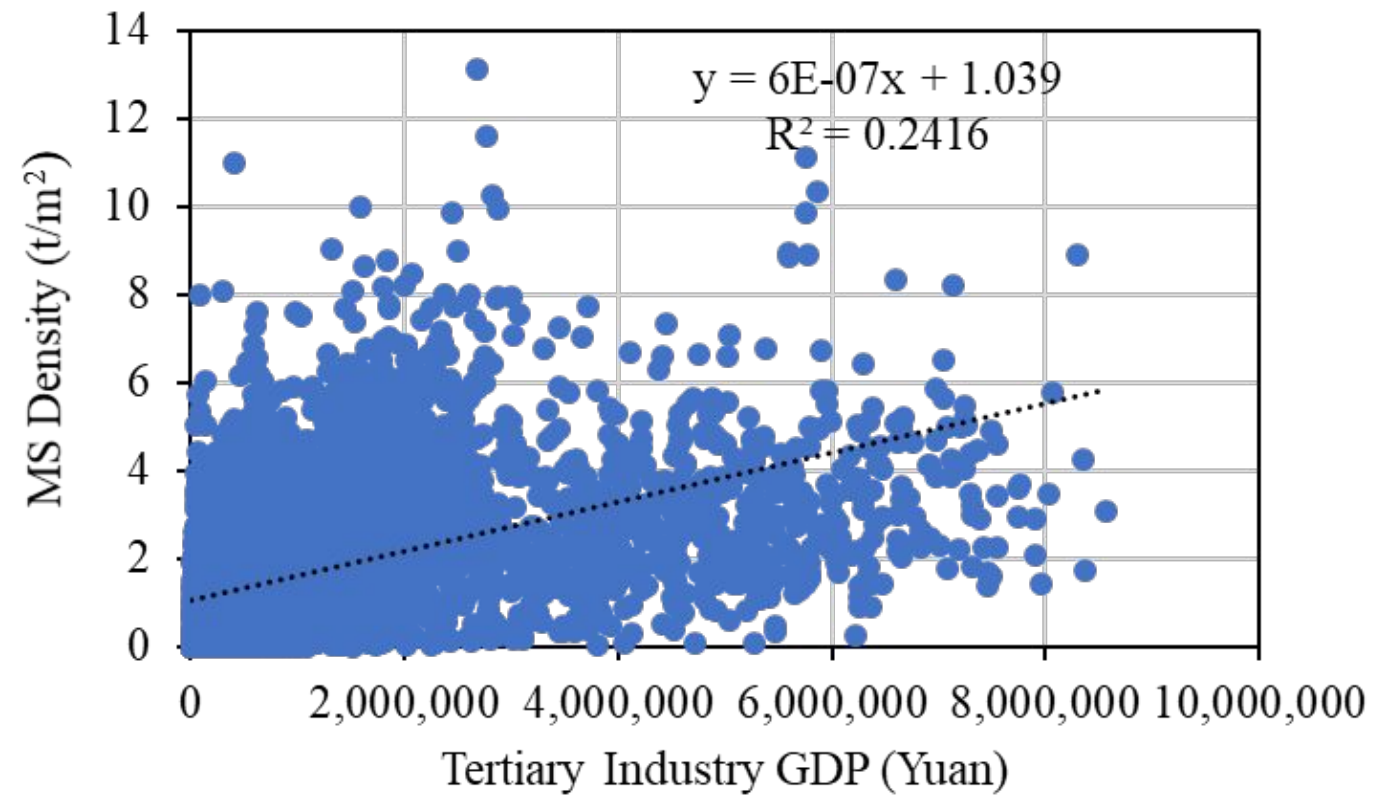

Figure S18. Correlation between Tertiary Industry GDP and built environment material stocks density in Beijing. 


\section{References in the supporting information:}

(1) Frischknecht, R.; Jungbluth, N.; Althaus, H. J.; Doka, G.; Dones, R.; Heck, T.; Hellweg, S.; Hischier, R.; Nemecek, T.; Rebitzer, G.; Spielmann, M. The Ecoinvent Database: Overview and Methodological Framework. Int. J. Life Cycle Assess. 2005, 10 (1), 3-9. https://doi.org/10.1065/lca2004.10.181.1.

(2) CLCD Database. 2015. IKE \& SCU-ISCP, 2015. Chinese Core Life Cycle Database Version 0.8. Available at eBalance 4.7 Software.

(3) Tanikawa, H.; Hashimoto, S. Urban Stock over Time: Spatial Material Stock Analysis Using 4d-GIS. Build. Res. Inf. 2009, 37 (5-6), 483-502. https://doi.org/10.1080/09613210903169394.

(4) Chen, C.; Shi, F.; Okuoka, K.; Tanikawa, H. The Metabolism Analysis of Urban Building by 4d-GIS - A Useful Method for New-Type Urbanization Planning in China. Univers. J. Mater. Sci. 2016, 4 (2), 40-46. https://doi.org/10.13189/ujms.2016.040204.

(5) Cheng, K.-L.; Hsu, S.-C.; Li, W.-M.; Ma, H.-W. Quantifying Potential Anthropogenic Resources of Buildings through Hot Spot Analysis. Resour. Conserv. Recycl. 2018, 133, 10-20. https://doi.org/10.1016/j.resconrec.2018.02.003.

(6) Marcellus-Zamora, K. A.; Gallagher, P. M.; Spatari, S.; Tanikawa, H. Estimating Materials Stocked by Land-Use Type in Historic Urban Buildings Using Spatio-Temporal Analytical Tools. J. Ind. Ecol. 2015, n/a-n/a. https://doi.org/10.1111/jiec.12327.

(7) Kleemann, F.; Lederer, J.; Rechberger, H.; Fellner, J. GIS-Based Analysis of Vienna's Material Stock in Buildings. J. Ind. Ecol. 2017, 21 (2), 368-380. https://doi.org/10.1111/jiec.12446.

(8) Miatto, A.; Schandl, H.; Forlin, L.; Ronzani, F.; Borin, P.; Giordano, A.; Tanikawa, H. A Spatial Analysis of Material Stock Accumulation and Demolition Waste Potential of Buildings: A Case Study of Padua. Resour. Conserv. Recycl. 2019, 142 (November 2018), 245-256. https://doi.org/10.1016/j.resconrec.2018.12.011.

(9) Stephan, A.; Athanassiadis, A. Quantifying and Mapping Embodied Environmental Requirements of Urban Building Stocks. Build. Environ. 2017, 114, 187-202. https://doi.org/10.1016/j.buildenv.2016.11.043.

(10) Han, J.; Chen, W.-Q.; Zhang, L.; Liu, G. Uncovering the Spatiotemporal Dynamics of Urban Infrastructure Development: A High Spatial Resolution Material Stock and Flow Analysis. Environ. Sci. Technol. 2018, 52 (21), 12122-12132. https://doi.org/10.1021/acs.est.8b03111.

(11) Mesta, C.; Kahhat, R.; Santa-Cruz, S. Geospatial Characterization of Material Stock in the Residential Sector of a Latin-American City. J. Ind. Ecol. 2018, 00 (0), 1-12. https://doi.org/10.1111/jiec.12723. 